\title{
Spinal subdural abscess following epidural steroid injection
}

\author{
Matthew J. Kraeutler, BS, Joseph D. Bozzay, MD, Matthew P. Walker, MD, and Kuruvilla John, MD \\ West Virginia University, Charleston Division, Charleston, West Virginia \\ The authors report the case of a 58 -year-old man who presented with a cervicothoracolumbosacral spinal subdural \\ abscess about a month after receiving an epidural steroid injection for management of low-back pain due to L5-S1 \\ disc herniation. Although he presented with symptoms concerning for a spinal etiology, the subdural empyema was not \\ evident on the initial MRI study and was observed on imaging 5 days later. This patient was successfully managed with \\ surgical intervention and antibiotic treatment, and he is doing well more than 21 months after the operation. It is possible \\ that a prior history of disc herniation or other spinal abnormality may increase a patient's risk of developing spinal subdu- \\ ral empyema. This case illustrates the risk of infection following spinal epidural steroid injections and the importance of \\ early recognition and intervention to successfully treat an extensive subdural abscess.
}

http://thejns.org/doi/abs/10.3171/2014.9.SPINE14159

KEY WORDS subdural abscess; subdural empyema; Staphylococcus aureus; epidural steroid injection; infection

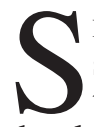
PINAL subdural abscess (SSA), also called a spinal subdural empyema, is extremely rare, with fewer than 100 cases reported. ${ }^{5}$ The low incidence of subdural empyema raises the importance of recognizing the characteristic signs and symptoms of these infections. To our knowledge, we report the second published case of a patient presenting with a spinal subdural empyema following a spinal epidural steroid injection.

\section{Case Report}

\section{History and Presentation}

A 58-year-old Caucasian man returned early from an overseas trip, complaining of increasing low-back pain, abdominal pain, constipation, and urinary retention lasting several days. He attributed these new symptoms to exacerbation of his disc herniation from lifting heavy luggage during his trip. He had a 4-year history of L5-S1 disc herniation with radiculopathy managed by transforaminal steroid injections every 6 months performed by an orthopedic spine specialist. His last transforaminal steroid injection was 24 days prior to presentation. This procedure was performed without complication under fluoroscopy. Transforaminal position was verified intraoperatively, and contrast was noted to track only in the epidural space. No perioperative antibiotics were given. Upon presentation, the patient denied intravenous drug use, fevers, chills, headache, nausea, or vomiting. He acknowledged perianal numbness, radiation of back pain to the posterior buttocks, and right leg weakness. His medical history was significant only for hypertension and hyperlipidemia.

A complete physical examination was unremarkable except for mild tenderness over the paraspinal muscles, mild abdominal tenderness to palpation, and a positive straight leg raise test on the right and a negative test on the left. No reflex spasticity or hypoactivity, clonus, or Babinski sign was observed.

Lumbar MRI without contrast (Fig. 1) showed slight improvement in the appearance of the patient's L5-S1 disc bulge compared with MRI performed 2 years previously, but persistent left neural foraminal narrowing and possible left S-1 nerve root impingement was still noted. No cauda equina or abscess was found. Abdominal radiography findings and complete blood count and basic metabolic panel were unremarkable, and urinary analysis showed no abnormality. The patient experienced relief with fentanyl and a Foley catheter, which drained $1 \mathrm{~L}$ of urine. He wanted to return home and follow up with a urologist and his orthopedic spine specialist as an outpa-

\section{ABBREVIATION SSA $=$ spinal subdural abscess .}

SUBMITTED February 8, 2014. ACCEPTED September 29, 2014.

INCLUDE WHEN CITING Published online October 24, 2014; DOI: 10.3171/2014.9.SPINE14159.

DISCLOSURE The authors report no conflict of interest concerning the materials or methods used in this study or the findings specified in this paper. 

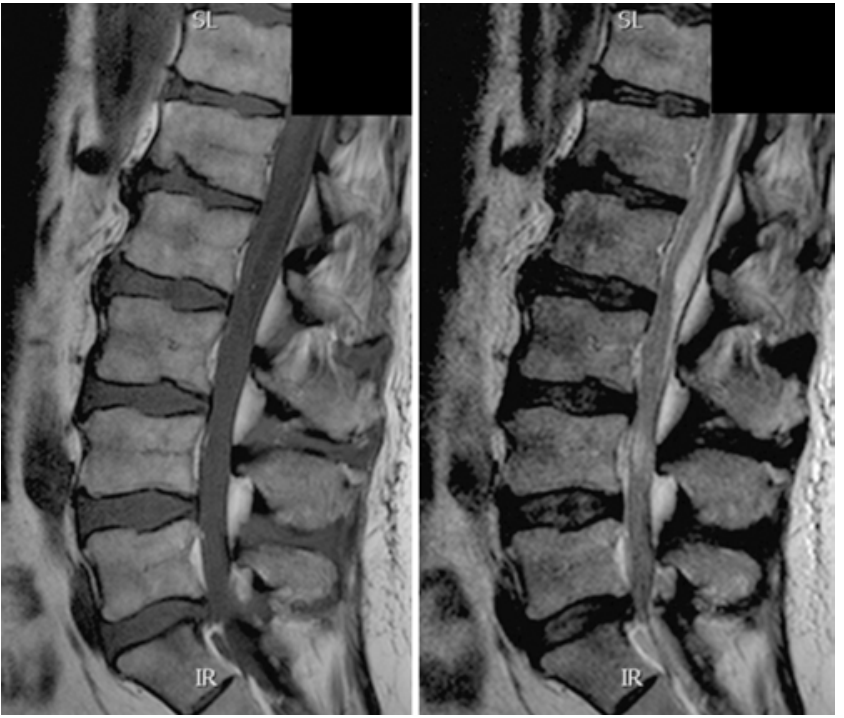

FIG. 1. Initial T1-weighted (left) and T2-weighted (right) sagittal MR images of the lumbar spine without contrast.

tient. He was discharged home with narcotics and a Foley catheter.

The patient visited his spine specialist 3 days later. He had experienced no improvement in his symptoms and noted that he had a fever the previous night. He complained of increasing low-back pain and right lower-extremity weakness. Physical examination revealed 2-3/5 strength throughout the right lower extremity with intact sensation. His left lower extremity had $4 / 5$ strength throughout. No reflex spasticity or hypoactivity, clonus, or Babinski sign was observed. A straight leg raise test was again positive on the right and negative on the left. He had pain to palpation over the lumbar spine. No swelling, edema, or erythema was noted over the spine. The rest of the physical examination was unremarkable.

Notable laboratory values included a white blood cell count of $13.2 \times 10^{3} / \mu 1$, blood $\mathrm{pH}$ of 7.50 , erythrocyte sedimentation rate of $60 \mathrm{~mm} / \mathrm{hr}$, and C-reactive protein of $144.3 \mathrm{mg} / \mathrm{L}$. His vital signs were notable for a temperature of $38.3^{\circ} \mathrm{C}$.

Cervical, thoracic, and lumbar MRI scans with and without gadolinium were obtained and showed an extensive subdural fluid collection extending from the cervical to sacral regions that enhanced after contrast administration (Fig. 2). There was evidence of extension to the basal meninges of the brain. These findings were concerning for a subdural abscess. A noncontrast head CT showed no abnormalities.

Retrospective review by another radiologist of the initial noncontrast MRI study performed 5 days previously failed to identify this abscess. However, posterior clumping for the rootlets was noted, raising the possibility of arachnoiditis. No contrast was administered with this initial MRI study, and thus no conclusion could be made with certainty.

\section{Operation}

The patient was started on intravenous vancomycin and ceftriaxone and was immediately taken to the operat-
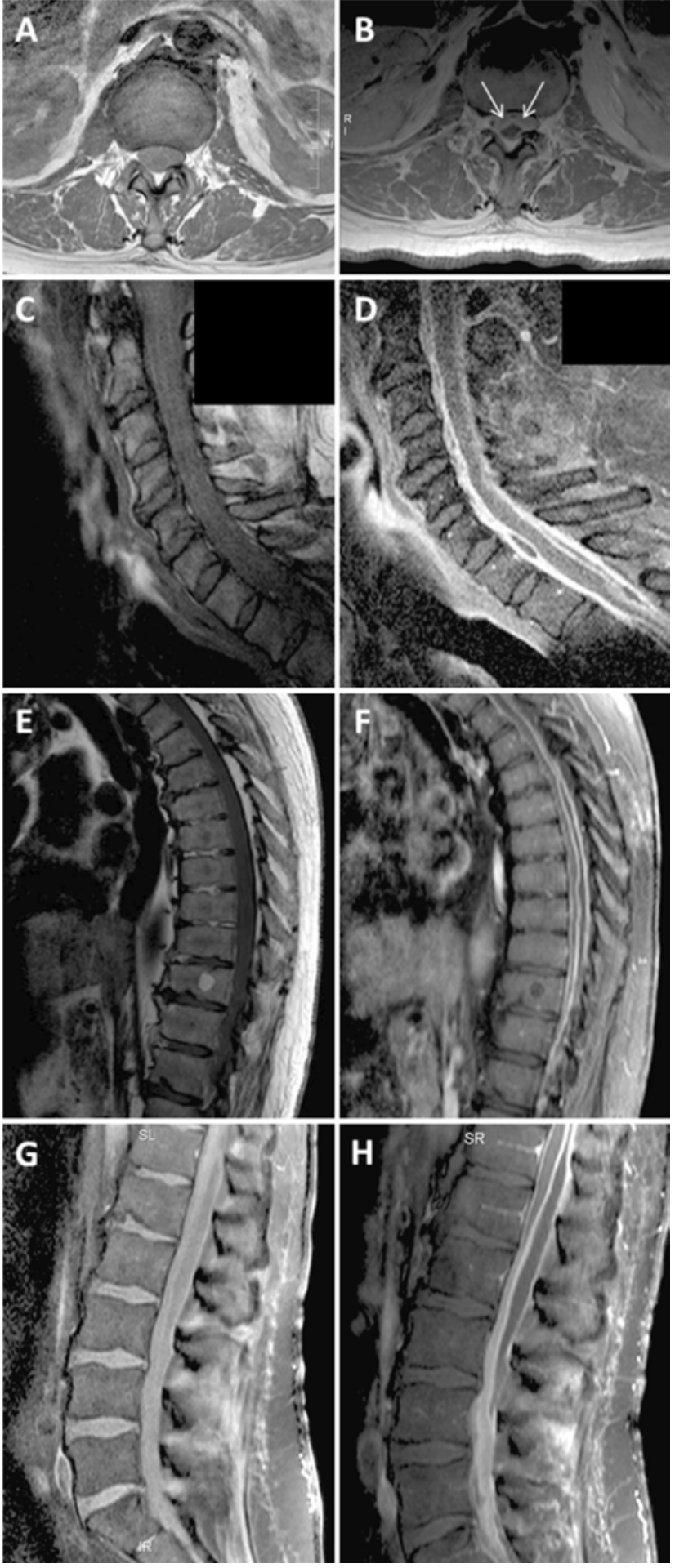

FIG. 2. A: Axial T1-weighted precontrast MR image of the L-2 vertebra. B: Axial T1-weighted postcontrast MR image showing subdural/ intradural fluid enhancement (arrows). C: Sagittal T1-weighted precontrast image of the cervical spine. D: Sagittal T1-weighted postcontrast image of the cervical spine. E: Sagittal T1-weighted precontrast image of thoracic spine. F: Sagittal T1-weighted postcontrast image of the thoracic spine, showing enhancement of the dura and an enhancing fluid collection displacing the spinal cord ventrally. G: Sagittal T1-weighted spectral presaturation with inversion recovery (SPIR) precontrast image of the lumbar spine. H: Sagittal T1-weighted SPIR postcontrast image of the lumbar spine. 
ing room for surgical decompression. After an L1-2 laminectomy, clear epidural space was exposed with a tense underlying dura. Thick, purulent fluid was immediately drained through a dural incision, and this fluid was sent for culture. The area was copiously irrigated with normal saline. The decision was made to leave the dura open so the fluid could continue to drain. A Hemovac drain was placed, and the fascia, subcutaneous tissue, and skin were subsequently closed with interrupted sutures. Culture of the fluid grew methicillin-sensitive Staphylococcus aureus. The steroid solution that was used for pain control prior to the patient's presentation in the emergency department came from a single-use vial and thus could not be tested for infection.

\section{Postoperative Course}

The total inpatient stay was 10 days. A peripherally inserted central catheter was placed, and the patient was treated with intravenous ceftriaxone as an outpatient for 42 days after surgery. His postoperative course was remarkable only for a urinary tract infection that was attributed to his Foley catheter. The patient's urinary retention, low-back pain, and right leg weakness improved within several weeks after the surgery, and he soon discontinued the Foley catheter and narcotics. At 12 and 19 months postoperatively, MRI showed marked resolution of the infection (Fig. 3). At 21 months postoperatively, the patient was examined in the office; his only complaint was persistent perianal numbness as well as poor sphincter control occasionally managed with laxatives.

\section{Discussion}

Spinal subdural abscess is a rare type of infection. Spinal epidural abscesses, while also rare, are much more common than spinal subdural empyema. ${ }^{5}$ To our knowledge, this is only the second case report of an SSA following an epidural steroid injection, and only the third report of an SSA following an epidural injection of any sort. Coumans and Walcott ${ }^{4}$ reported the case of a 53-year-old
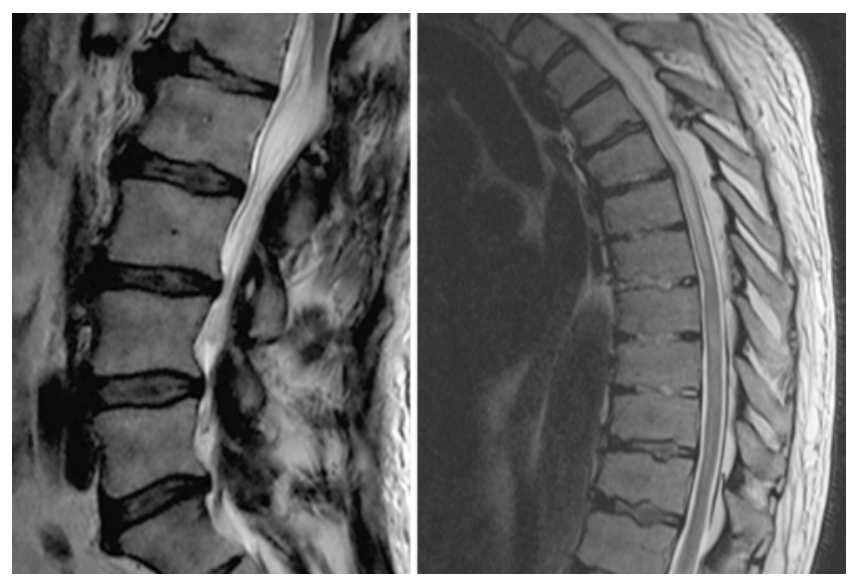

FIG. 3. Left: Sagittal T2-weighted MR image obtained 12 months postoperatively, showing resolution of the infection with herniation of the dural sac through the laminectomy defect. Right: Sagittal T2-weighted MR image obtained 19 months postoperatively, showing marked resolution of the subdural abscess. man who received a thoracic epidural injection at the T-7 level for treatment of thoracic back pain 11 weeks prior to presentation in the emergency department with lumbar pain and fever. In addition, this patient also received an acromial bursal injection 2 days prior to his presentation. Blood cultures grew methicillin-sensitive $S$. aureus and, following an L-2 laminectomy, a yellow fluid was obtained from the subdural space, which was cultured but did not grow any bacteria after 7 days. Unlike our patient, this patient did not have a prior history of disc herniation as far as we know.

Another case report involved a 38-year-old woman in advancing labor who received a nonsteroidal epidural injection at L2-3 consisting of $0.1 \%$ bupivacaine and fentanyl $2 \mu \mathrm{g} / \mathrm{ml} .{ }^{3}$ An epidural catheter was also placed. The patient received a total of 3 epidural top-ups over the next few hours. Seven days following the injection, the patient was readmitted with severe back pain and a mildly elevated white blood cell count. MRI showed a subdural abscess from T-10 to L-2, for which the patient underwent an L2-3, L1-2 laminectomy. Cultures from the abscess grew methicillin-sensitive $S$. aureus.

In the present case, the source of the infection was likely skin contamination from the patient. However, it cannot be ruled out that the steroid solution was the initial source of infection, especially considering the recent outbreak of fungal infections due to contaminated methylprednisolone acetate solutions. ${ }^{7}$ It is likely that the infection spread from the epidural to the subdural space.

The most common signs and symptoms of spinal subdural abscess include fever, back pain, and bladder dysfunction, as our patient presented with, as well as para-/ tetraparesis and disturbances of consciousness. ${ }^{8,12}$ In the majority of cases, these infections occur in the thoracolumbar region ${ }^{12}$ and are due to $S$. aureus., ${ }^{1,211-14}$ Mycobacterium tuberculosis has also been reported as an infectious agent involved in SSA..$^{910}$ Surgical drainage, antibiotics, and, in some cases, laminectomy are necessary for appropriate management. Patients should be treated immediately, as neurological deficits may progress quickly and death has been reported as a result of SSA.

A review of spinal epidural abscess in 43 patients found that most patients had underlying conditions predisposing to infection, including spinal abnormality or trauma. ${ }^{6}$ Another review, ${ }^{12}$ focusing on SSA, supports this finding, citing degenerative joint disease, trauma, surgery, and drug injections as conditions predisposing to infection. Thus, our patient's prior history of an S-1 disc herniation may have predisposed him to a subdural empyema. It is possible that the patient had experienced an intradural disc herniation, which may have allowed the infection to spread from the epidural to the subdural space. Most intradural disc herniations occur in the lumbar region, with $10 \%$ occurring between L5-S1. ${ }^{6}$

Patients undergoing spinal epidural steroid injections may be at increased risk for subdural empyema. Utmost attention should be directed at a possible infectious process when a patient presents with symptoms concerning for spinal etiology following an epidural injection. An MRI study with contrast should be ordered to catch the early manifestations of a spinal abscess. Further research 
may be helpful to determine the incidence of spinal subdural empyema following epidural injections, as well as to determine if patients with a prior history of disc herniation are at increased risk of spinal subdural empyema.

\section{References}

1. Chen MH, Chen MH, Huang JS: Cervical subdural empyema following acupuncture. J Clin Neurosci 11:909-911, 2004

2. Chern SH, Wei CP, Hsieh RL, Wang JL: Methicillin-resistant Staphylococcus aureus retropharyngeal abscess complicated by a cervical spinal subdural empyema. J Clin Neurosci 16:144-146, 2009

3. Collis RE, Harries SE: A subdural abscess and infected blood patch complicating regional analgesia for labour. Int J Obstet Anesth 14:246-251, 2005

4. Coumans JV, Walcott BP: Rapidly progressive lumbar subdural empyema following acromial bursal injection. J Clin Neurosci 18:1562-1563, 2011

5. Darouiche RO: Spinal epidural abscess and subdural empyema. Handb Clin Neurol 96:91-99, 2010

6. Darouiche RO, Hamill RJ, Greenberg SB, Weathers SW, Musher DM: Bacterial spinal epidural abscess. Review of 43 cases and literature survey. Medicine (Baltimore) 71:369385, 1992

7. Kuehn BM: CDC probes new outbreak associated with compounded steroids. JAMA 309:2541, 2013

8. McCabe JJ, Murphy RP: Spinal subdural abscess. JAMA Neurol 70:266-267, 2013

9. Mikić D, Roganović Z, Culafić S, Dimitrijević RR, Begović V, Milanović M: Subdural tuberculous abscess of the lumbar spine in a patient with chronic low back pain. Vojnosanit Pregl 69:1109-1113, 2012

10. Shukla D, Gangadharan J, Devi BI, Ambekar S: Tuberculous spinal subdural abscess in an infant with dermal sinus. Neurol India 60:236-237, 2012

11. Sorar M, Er U, Seçkin H, Ozturk MH, Bavbek M: Spinal subdural abscess: a rare cause of low back pain. J Clin Neurosci 15:292-294, 2008

12. Velissaris D, Aretha D, Fligou F, Filos KS: Spinal subdural staphylococcus aureus abscess: case report and review of the literature. World J Emerg Surg 4:31, 2009

13. Vural M, Arslantaş A, Adapinar B, Kiremitçi A, Usluer G, Cuong B, et al: Spinal subdural Staphylococcus aureus abscess: case report and review of the literature. Acta Neurol Scand 112:343-346, 2005

14. Wu AS, Griebel RW, Meguro K, Fourney DR: Spinal subdural empyema after a dural tear. Case report. Neurosurg Focus 17(6):E10, 2004

\section{Author Contributions}

Drafting the article: Kraeutler. Critically revising the article: Bozzay, Walker, John. Reviewed submitted version of manuscript: all authors. Approved the final version of the manuscript on behalf of all authors: Kraeutler. Study supervision: John.

\section{Supplemental Information}

\section{Previous Presentation}

Portions of this work were presented in poster form at the American College of Physicians 2013 West Virginia Chapter Scientific Meeting, Roanoke, WV, October 19, 2013.

\section{Correspondence}

Matthew J. Kraeutler, Robert C. Byrd Health Sciences Center, Charleston Division, 3110 MacCorkle Ave. SE, Charleston, WV 25304. email: mkraeutl@mix.wvu.edu. 